\title{
DIFERENTES FONTES DE LIPÍDIOS TESTADAS NA CRIAÇÃO DE LARVAS DE JUNDIÁ (Rhamdia quelen), PISCES, PIMELODIDAE ${ }^{1}$
}

\author{
DIFFERENT SOURCES OF FAT TESTED ON LARVAE CULTURE OF JUNDIA \\ (Rhamdia quelen), PISCES, PIMELODIDAE
}

\section{Oclecio Uliana ${ }^{2}$ José Henrique Souza da Silva $^{3}$ João Radünz Neto ${ }^{4}$}

\section{RESUMO}

O presente trabalho foi desenvolvido para avaliar a influência de diferentes fontes de lipídios sobre o desempenho de larvas de jundiá (Rhamdia quelen). Foi realizado no Setor de Piscicultura do Departamento de Zootecnia da Universidade Federal de Santa Maria, utilizando-se um sistema de criação de larvas com recirculação d'água, termorregulada. $O$ experimento teve duração de 21 dias, testando-se 7 tratamentos com três repetições. Testaram-se diferentes fontes de lipídios: óleo de canola, óleo de soja, óleo de fígado de bacalhau, óleo de girassol e óleo de milho, incorporados na proporção de $5 \%$ de uma dieta base (fígado e levedura). O tratamento com óleo de canola resultou em $94 \%$ de sobrevivência e larvas com 19,97mm de comprimento total e $54,3 \mathrm{mg}$ de peso médio. De acordo com os resultados obtidos no experimento, conclui-se que os óleos de canola e fígado de bacalhau são mais eficientes como suplemento lipídico na nutrição de larvas de jundiá (Rhamdia quelen).

Palavras-chave: lipídios, fígado, larva, levedura, sobrevivência, Rhamdia quelen.

\section{SUMMARY}

The present study was developed in order to evaluate the influence of different sources of fat on the Rhamdia quelen larvae performance. It was carried out at the Animal Husbandry Department - Fish culture Section of the Federal University of Santa Maria, using a system of larvae culture with thermoregulation and recirculation of water. There were seven (7) treatments, with three replicates each one and the different sources of fat tested during 21 days were: canola oil, soybean oil, cod liver oil, sunflower oil or corn oil incorporated in the rate of $5 \%$ of a basal diet (liver and yeast). The treatment with canola oil resulted in a $94 \%$ survival rate, and larvae with $19.97 \mathrm{~mm}$ of total length and 54,3mg of individual body weight. According to the results obtained in the experiment, it was concluded that canola and cod liver oil are more efficient as fat supplement in Rhamdia quelen larvae nutrition.

Key words: lipids, liver, larvae, yeast, survival, Rhamdia quelen.

\section{INTRODUÇÃO}

O jundiá (Rhamdia quelen) é uma das espécies nativas do Rio Grande do Sul que se destacam como uma das mais promissoras. Segundo LUCHINI \& AVENDAÑO (1985), o jundiá é um peixe de rápido crescimento, com fácil adaptação à criação intensiva, rústico, facilmente induzida à reprodução, com alta taxa de fecundação, possuindo ainda carne saborosa com baixo teor de gordura e com poucas espinhas.

Atualmente, a produção de alevinos de jundiá tem aumentado e um dos principais problemas na produção é a baixa sobrevivência ao final do estádio larval, sendo que o mercado para essa espécie é ascendente. A criação de larvas é um processo delicado tanto em espécies de peixes de água doce como salgada, pois é o período mais sensível na vida dos peixes. As dificuldades são devido, principalmente, ao pequeno tamanho inicial das larvas (alguns milímetros de comprimento) que começam a se alimentar logo após absorção do saco vitelino. Os alimentos usados, nessa fase de vida, devem conter fontes protéicas de alta digestibilidade e atratopalatabilidade, proporcionando a ingestão de um

\footnotetext{
${ }^{1}$ Parte da Dissertação de Mestrado do primeiro autor apresentada ao Curso de Pós-graduação em Zootecnia, Universidade Federal de Santa Maria (UFSM)

${ }^{2}$ Engenheiro Agrônomo, Mestre em Zootecnia.

${ }^{3}$ Engenheiro Agrônomo, PhD., Professor Adjunto do Departamento de Zootenia, UFSM.

${ }^{4}$ Engenheiro Agrônomo, Doutor, Professor Adjunto do Departamento de Zootecnia, UFSM, 97105-900 Santa Maria, RS, Bolsista CNPq, E-mail: radunz@ccr.ufsm.br. Autor para correspondência.
} 
alimento equilibrado com todos os ingredientes necessários ao desenvolvimento larval.

Para algumas espécies de água doce, já existem, atualmente, alimentos artificiais capazes de assegurar a sobrevivência e o crescimento de larvas, dispensando o uso de alimentos vivos (Artemia salina e Brachionus plicatilis) como é o caso da carpa (Cyprinus carpio) (BERGOT et al., 1986 e RADÜNZ NETO, 1993), do coregonus (Coregonus lavaretus maraena) (CHARLON et al., 1986), e do próprio jundiá (Rhamdia quelen) (PIAIA, 1996 e FONTINELLI, 1997). PIAIA (1996) encontrou bons resultados na larvicultura do jundiá usando dietas preparadas com fígado bovino e levedura de cana, sem a adição de lipídios. No entanto, além da fração protéica, o lipídio é importante fonte energética em rações usadas na alimentação de larvas, fornecendo muitos ácidos graxos essenciais (AGE) de elevado valor energético. Alguns estudos foram realizados por RADÜNZ NETO (1993), SATOH $\boldsymbol{e t}$ al. (1989a) e LEGENDRE $\boldsymbol{e t}$ al. (1995) avaliando a sobrevivência, crescimento e conversão do alimento nas fases iniciais de diversas espécies de peixes (carpa, bagres norte-americano e africano, respectivamente) testando diferentes fontes de lipídios e salientando a importância dos lipídios e de ácidos graxos das séries (n-3) e (n-6) nas dietas dos peixes. SATOH et al. (1989b) constataram que alevinos de bagre norteamericano desenvolveram-se bem com dietas contendo $2 \%$ de ácido linolênico (18:2 n-3), tanto quanto com aquelas contendo $2,5 \%$ de óleo de fígado de bacalhau, mais $2,5 \%$ de óleo de milho.

Assim, o objetivo do trabalho foi avaliar a influência de diferentes fontes de lipídios visando a melhorar os índices de sobrevivência e ganho de peso de larvas de jundiá em criações intensivas.

\section{MATERIAL E MÉTODOS}

O trabalho teve duração de 21 dias e foi realizado no Setor de Piscicultura do Departamento de Zootecnia do Centro de Ciências Rurais da Universidade Federal de Santa Maria durante o mês de outubro de 1996

O mesmo foi conduzido num sistema de criação de larvas com recirculação d'água, termorregulada, conforme o proposto por CHARLON \& BERGOT (1984), com 21 unidades experimentais, e um reservatório com capacidade de 1000 litros, dotado de um filtro biológico. Cada unidade experimental era constituída de duas bacias plásticas, sendo a interna com dimensão de 33 x 19 x $10 \mathrm{~cm}$, de formato retangular. Numa das extremidades, tem uma abertura protegida por uma tela com abertura de malha de $300 \mu$, permitindo a renovação da água. A bacia externa com dimensão de 34 x 23 x $11 \mathrm{~cm}$, de mesmo formato, possui um dreno lateral, permitindo manter o nível da água da bacia interna.

A água utilizada no sistema de criação era proveniente de um poço artesiano e a temperatura foi mantida entre $23^{\circ} \mathrm{C} \pm 1$ durante todo o experimento. A vazão utilizada nas unidades de criação foi de $0,15 \ell / \mathrm{min}$ na primeira semana, $0,25 \ell / \mathrm{min}$ na segunda semana e $0,40 \mathrm{l} / \mathrm{min}$ na terceira semana experimental. As análises da água do sistema de criação foram realizadas diariamente verificando-se os níveis de oxigênio (maior que 7,5 $\pm 1 \mathrm{mg} / \ell$ ), com o pH estável $(7,5)$, com os níveis de amônia e nitrito mantendo-se dentro de níveis aceitáveis ao longo do experimento.

As larvas de jundiá utilizadas no experimento foram obtidas através de indução à reprodução de reprodutores da própria instituição. Após a eclosão, as larvas permaneceram em incubadoras do tipo ZUG, a uma temperatura de $23^{\circ} \mathrm{C}$, durante três dias para absorção do saco vitelino, sendo que, nesse período, as mesmas não receberam alimentação. Foram então contadas de dez em dez, ao acaso, e distribuídas no sistema de criação até totalizar um número de 160 larvas por unidade experimental.

A composição das rações utilizadas no experimento encontra-se descrita na tabela 1. A composição centesimal das fontes protéicas usadas, expressa em matéria parcialmente seca é: levedura (Umidade: $6,35 \% ; \mathrm{PB}=38 \% ; \mathrm{EE}=0,35 \% ; \mathrm{FB}=$ $0,75 \% ; \mathrm{MM}=6,98 \%$ ) e do fígado bovino (Umidade: $7,03 \% ; \mathrm{PB}=66,38 \% ; \mathrm{EE}=12,94 \% ; \mathrm{FB}=0,0 \%$; $\mathrm{MM}=4,44 \%$ ). Os alimentos foram preparados no próprio setor de piscicultura da instituição, conforme metodologia descrita a seguir: - A levedura de cana foi previamente moída (moinho a martelo laboratorial) e peneirada em malha de $75 \mu$, utilizando-se a

Tabela 1 - Composição das rações experimentais (A) utilizadas para larvas de jundiá (Rhamdia quelen), em percentagem.

$\begin{array}{llllllll}\text { INGREDIENTES } & \text { A1 } & \text { A2 } & \text { A3 } & \text { A4 } & \text { A5 } & \text { A6 } & \text { A7 }\end{array}$

$\begin{array}{lrrrrrrr}\text { Fígado bovino (mat.seca)* }^{*} & 34 & 34 & 34 & 34 & 34 & 34 & 34 \\ \text { Levedura de cana }^{*} & 50 & 50 & 50 & 50 & 50 & 50 & 50 \\ \text { Premix vitamínico* } & 10 & 10 & 10 & 10 & 10 & 10 & 10 \\ \text { Premix mineral* }^{*} \text { Óleo de canola } & 1 & 1 & 1 & 1 & 1 & 1 & 1 \\ \text { Óleo de girassol } & 5 & - & - & - & - & - & - \\ \text { Óleo de soja } & - & 5 & - & - & - & - & - \\ \text { Óleo de fígado de bacalhau } & - & - & 5 & 2,5 & - & - & - \\ \text { Óleo de milho } & - & - & - & 2,5 & 5 & 2,5 & - \\ & - & - & - & - & - & 2,5 & 5\end{array}$

\footnotetext{
* Segundo FONTINELLI (1997).
} 
fração granulométrica inferior para o preparo das rações; e o fígado bovino cru ( $66 \%$ de umidade) foi moído em máquina de moer carne elétrica para facilitar a mistura dos ingredientes. A preparação dos alimentos constituiu-se, inicialmente, na mistura da levedura e fígado bovino cru junto com as vitaminas, lipídios e minerais até completa homogeneização. No preparo das rações, não foi necessário a adição de água. Cada ração foi então colocada na máquina de moer carne formando "pelets", para facilitar a secagem e conservação da ração. $O$ processo de secagem foi realizado ao sol no período das 10 às 16 horas, a uma temperatura de $45^{\circ} \mathrm{C} \pm 3^{\circ} \mathrm{C}$. Completado o período de secagem, as dietas foram novamente moídas e peneiradas, separando-se então as diferentes granulometrias, entre 100 a 200, 200 a 400 e 400 a $600 \mu$ utilizadas na alimentação durante a primeira, segunda e terceira semanas experimentais, respectivamente (PIAIA, 1996 e FONTINELLI, 1997). As rações foram acondicionadas em sacos plásticos e armazenadas sob refrigeração a $4^{\circ} \mathrm{C}$ para melhor conservação.

A partir do dia seguinte à estocagem das larvas nas unidades experimentais, as mesmas foram alimentadas através de um alimentador automático controlado por um temporizador, o qual fornecia, a cada meia hora, pequena porção de ração, de modo a formar uma delgada camada flutuante sobre a superfície da água. Essa distribuição era feita ao longo de 14 horas diárias (das 7:00 às 21:00 horas). Os distribuidores de alimento foram reabastecidos a cada três dias, com uma pequena porção de ração $(3 \mathrm{~g}$ por unidade), sendo que a sobra existente no alimentador era descartada.

Diariamente, foi realizada a limpeza das bacias através de transferência das larvas para bacias limpas, sendo contabilizada a mortalidade. No final do dia, as unidades experimentais eram sifonadas, utilizando-se mangueira plástica de $0,4 \mathrm{~cm}$ de diâmetro, para retirada do excesso de alimento e resíduos acumulados no fundo das bacias. Semanalmente, foi realizada a biometria de um grupo de 10 larvas por unidade experimental. As medidas de comprimento total e comprimento padrão de cada larva foram tomadas com o auxílio de lupa e papel milimetrado. Para realizar esse processo, as mesmas eram tranqüilizadas com tri-fenoxietanol na concentração de $0,15 \mathrm{ppm}$.

No final do período experimental, os peixes sobreviventes em cada unidade experimental foram submetidos à contagem, medição e pesagem a qual foi realizada utilizando-se uma balança ACATEC (precisão de 0,01g).

Os parâmetros avaliados foram: - Comprimento total (CT): (medida entre a região anterior da cabeça até o final do lóbulo superior da nadadeira caudal) no início do experimento, aos 7, 14 e 21 dias; - Comprimento padrão (CP) (medida entre a região anterior da cabeça até o início da nadadeira caudal), no início do experimento, aos 7, 14 e 21 dias; - Peso médio (PM) é a média do valor calculado a partir do peso total das larvas da unidade experimental, dividido pelo número de peixes sobreviventes no final do experimento; - Biomassa: avaliada através do produto do peso médio versus sobrevivência, aos 21 dias experimentais; - Taxa de crescimento específico (TCE), sendo que este índice foi calculado através de uma fórmula deduzida matematicamente, segundo LEGENDRE et al. (1995): TCE $=100(\ln$ Peso2 -ln Peso1) / t onde: ln = logaritmo neperiano; Peso2 $=$ peso médio individual das larvas obtido ao final do experimento; Peso1 = peso médio individual das larvas obtido no início do experimento; $\mathrm{t}$ = tempo de realização do experimento, em dias; - Índice de Mortalidade Real, verificado ao final do experimento, através da contabilização do número de sobreviventes finais.

O delineamento experimental foi inteiramente casualizado, com sete tratamentos e três repetições, sendo que cada bacia constituía a unidade experimental. Os dados obtidos no experimento foram submetidos à análise de variância e teste $\mathrm{F}$ ao nível de 5\% de significância e foi aplicado o teste de Tukey para comparação entre as médias dos tratamentos no mesmo nível de significância. Usou-se o programa SAS (1995), versão 6.08, instalado no sistema operacional CMS, no Centro de Processamento de Dados da UFSM.

\section{RESULTADOS E DISCUSSÃO}

Os resultados de sobrevivência deste experimento (Tabela 2) mostraram índices significativos $(\mathrm{P}<0,05)$ para os tratamentos com óleo de soja e óleo de fígado de bacalhau, respectivamente. As taxas de sobrevivência foram superiores às encontradas por PIAIA \& RADÜNZ NETO (1997), usando a mesma dieta base (fígado, levedura), em trabalho com larvas de jundiá, e aos valores obtidos por LEGENDRE $\boldsymbol{e t}$ al. (1995), com larvas de bagre africano aos 15 dias de alimentação, quando testaram diferentes fontes de lipídios incorporadas em dieta à base de fígado bovino $(30 \%)$ e levedura (50\%).

Os resultados de comprimento total obtidos aos 21 dias apresentaram valores significativos $(\mathrm{P}<0,05)$ nos tratamentos com óleo de canola e óleo de fígado de bacalhau (Tabela 2) e foram superiores aos citados por PIAIA (1996) que obteve $16,46 \mathrm{~mm}$ de comprimento total, usando as mesmas fontes 
Tabela 2 - Comprimento total (CT), peso médio (PM), sobrevivência (S), biomassa (P x S) e taxa de crescimento específico (TCE) das larvas de jundiá ao final do experimento.

\begin{tabular}{cccccc}
\hline T & CT (mm) & PM (mg) & S (\%) & P x S & TCE (\%/dia) \\
\hline & & & & & \\
A1 & $19,97^{\mathbf{a}}$ & $54,28^{\mathbf{a}}$ & $93,57^{\mathbf{a b}}$ & $50,73^{\mathbf{a}}$ & $15,71^{\mathbf{a}}$ \\
A2 & $16,26^{\mathbf{b}}$ & $38,51^{\mathbf{c}}$ & $88,80^{\mathbf{b}}$ & $34,18^{\mathbf{c}}$ & $14,06^{\mathbf{c}}$ \\
A3 & $17,08^{\mathbf{b}}$ & $45,60^{\mathbf{a b c}}$ & $95,23^{\mathbf{a}}$ & $43,40^{\mathbf{a b}}$ & $14,88^{\mathbf{a b c}}$ \\
A4 & $16,06^{\mathbf{b}}$ & $41,85^{\mathbf{b c}}$ & $88,56^{\mathbf{b}}$ & $37,04^{\mathbf{b c}}$ & $14,48^{\mathbf{b c}}$ \\
A5 & $19,91^{\mathbf{a}}$ & $51,12^{\mathbf{a b}}$ & $94,51^{\mathbf{a}}$ & $48,79^{\mathbf{a}}$ & $15,42^{\mathbf{a b}}$ \\
A6 & $15,51^{\mathbf{b}}$ & $41,27^{\mathbf{c}}$ & $91,18^{\mathbf{a b}}$ & $37,63^{\mathbf{b c}}$ & $14,41^{\mathbf{c}}$ \\
A7 & $15,33^{\mathbf{b}}$ & $44,65^{\mathbf{b c}}$ & $88,14^{\mathbf{b}}$ & $39,58^{\mathbf{b c}}$ & $14,77^{\mathbf{a b c}}$ \\
& & & & & \\
\hline
\end{tabular}

Médias seguidas por letras diferentes, na coluna, apresentam diferenças significativas pelo teste de Tukey $(\mathrm{p}<0,05)$.

protéicas (fígado e levedura). Os outros tratamentos testados não diferiram significativamente entre si. As médias de comprimento padrão acompanharam as de comprimento total quanto às diferenças estatísticas. Esse parâmetro é importante uma vez que poderá indicar se ocorreu algum problema de má formação da nadadeira caudal, o que não foi constatado neste trabalho. Os resultados apresentados na tabela 2 permitem verificar que aos 21 dias as larvas de jundiá, alimentadas com dietas suplementadas com óleo de canola ou óleo de fígado de bacalhau, apresentaram melhor crescimento. Esses óleos apresentam maior presença de ácidos graxos da série (n3) (NRC, 1993) os quais são considerados essenciais para o bagre norte-americano (SATOH et al., 1989a). No presente experimento, a substituição parcial $(50 \%)$ dos óleos de soja e milho por óleo de fígado de bacalhau (tratamentos A4 e A6, respectivamente) prejudicou o crescimento das larvas de jundiá.

O peso médio foi significativamente $(\mathrm{P}<0,05)$ mais elevado nas larvas suplementadas com óleo de canola do que naqueles que receberam óleo de girassol, óleo de soja + óleo de fígado de bacalhau, óleo de fígado de bacalhau + óleo de milho e somente óleo de milho. Também pode-se observar que os tratamentos com óleo de girassol e uma mistura composta por óleo de milho + óleo de fígado de bacalhau foram os que apresentaram os menores pesos. Por outro lado, SATOH et al. (1989b), em estudos realizados com alevinos de bagre norte americano, comprovaram que a mistura $2,5 \%$ de óleo de fígado de bacalhau $+2,5 \%$ de óleo de milho em dieta purificada (caseína e dextrina) apresentaram resultados superiores em ganho de peso, quando comparados ao tratamento de mesma dieta base, porém com $5 \%$ de óleo de fígado de bacalhau. Já SZLAMINSKA et al. (1990) utilizaram uma dieta com $50 \%$ de levedura e $35 \%$ de fígado bovino, sem suplementação de óleo, e obtiveram $262 \mathrm{mg}$ de peso aos 21 dias para larvas de carpa comum (Cyprinus carpio).

Os maiores valores de $\mathrm{P}$ x $\mathrm{S}$ obtidos para o experimento foram da ordem de 50,73 e 48,79 para os tratamentos com óleo de colza e com óleo de fígado de bacalhau respectivamente, e não diferiram significativamente entre si, conforme indicado na tabela 2.

A maior taxa de crescimento específico (15,71\%/dia) foi encontrada com o tratamento contendo óleo de canola, mas não apresentou diferença significativa dos tratamentos A3, A5 e A7 (Tabela 2). LEGENDRE $\boldsymbol{e t}$ al. (1995), utilizando fígado e levedura (30 e $50 \%$, respectivamente) com $7,5 \%$ de óleo de fígado de bacalhau obtiveram $24,5 \%$ dia como valor de taxa de crescimento específico para larvas de bagre africano aos 15 dias experimentais. Os tratamentos com óleo de girassol e óleo de fígado de bacalhau + óleo de milho, apresentaram as menores taxas de crescimento (14,06 e 14,41, respectivamente). Esses valores ainda são baixos e podem ser melhorados, já que HEPHER (1988) salienta que os peixes podem aumentar cerca de $40 \%$ ou mais do seu peso / dia, no caso de larvas, diminuindo a velocidade de crescimento relativo com o desenvolvimento do peixe (menos de $1 \%$ do seu peso/ dia em peixes com mais de $1 \mathrm{~kg}$ ).

\section{CONCLUSÕES}

Através dos resultados obtidos, conclui-se que todos os tratamentos permitem uma ótima sobrevivência, e que os tratamentos com óleo de canola e de figado de bacalhau permitem maior crescimento das larvas de jundiá.

\section{REFERÊNCIAS BIBLIOGRÁFICAS}

BERGOT, P., CHARLON, N., DURANTE, H. The effect of compound diets feeding on growth and survival of coregonid larvae. Arch Hydrobiol Beich n.22, p.265-272, 1986.

CHARLON, N., BERGOT, P. Rearing system for feeding fish larvae on dry diets. Trial with carp (Cyprinus carpio) larvae. Aquaculture, n.41, p.1-9, 1984

CHARLON, N., DURANTE, H., ESCAFFRE, A.M., $\boldsymbol{e} \boldsymbol{t} \boldsymbol{a l}$. Alimentation artificiel des larves de carpe (Cyprinus carpio). Aquaculture, n.54, p.83-88, 1986.

FONTINELLI, E. Efeitos do uso do concentrado protéico de soja, com e sem suplementação de aminoácidos, sobre o crescimento e sobrevivência das larvas de jundiá (Rhamdia quelen). Santa Maria, RS, 1997. 38p. Dissertação (Mestrado em Zootecnia) - Curso de Pós-graduação em Zootecnia - Universidade Federal de Santa Maria, 1997. 
HEPHER, B. Nutrition of pond fishes. Melbourne, Austrália: Cambridge University, 1988. 388p.

LEGENDRE, M., KERDCHUEN, N., CORRAZE, G., $\boldsymbol{e}$ t $\boldsymbol{a l}$. Larval rearing of on African catfish Heterobranchus longifilis (Teleostei, Clariidae): effect of dietary lipids on growth, survival and fatty acid composition of fry. Aquat Living Resour n.8, p.363-365, 1995.

LUCHINI, L., AVENDAÑO, T. Primeros resultados de cultivo de un pez de aguas calidas (Rhamdia sapo) con fines de producion y consumo humano. Rev Arg Prod Anim, v.4, n.5, p.621-629, 1985

NATIONAL RESEARCH COUNCIL (NRC). Nutrient requeriments of warmwater fishes. Washington, $\mathrm{DC}$ : National Academy of Sciences, 1993. 114p.

PIAIA, R. Efeito do uso de diferentes fontes protéicas e diferentes níveis de proteína sobre o crescimento e sobrevivência de larvas de jundiá (Rhamdia quelen). Santa Maria, RS, 1996. 48p. Dissertação (Mestrado em Zootecnia) - Curso de Pós-graduação em Zootecnia - Universidade Federal de Santa Maria, 1996.

PIAIA, R., RADÜNZ NETO, J. Efeito de níveis crescentes de levedura de álcool em rações contendo fígado bovino sobre a performance das larvas de jundiá (Rhamdia quelen). Ciência Rural, v.27, n.2, p.313-317, 1997.

RADÜNZ NETO, J. Détermination des besoins nutritionnels en acides gras essentiels chez les larves de carpe (Cyprinus carpio). Bordeaux, França, 1993. 121p. Tese (Doctorat en Sciences des Aliments) - Ecole Doctorale des Sciences Biologiques et Medicales, Université de Bordeaux I, 1993.

SAS. Statistical Analisis System. User's Guide. Version 6,08, 4 ed. North Caroline : SAS Institute, 1995. 846p.

SATOH S., POE, W.E., WILSON, R.P. Studies on the essential fatty acid requeriment of channel catfish, Ictalurus punctatus. Aquaculture, n.79, p.121-128, 1989a.

SATOH S., POE, W.E., WILSON, R.P. Effect of dietary n-3 fatty acids on weight gain and liver polar lipid fatty acid composition of fingerling Channel catfish. J Nutrition, n.119, p.23-28, 1989 b.

SZLAMINSKA, M., ESCAFFRE, A.M., ALAMI-DURANTE, H. et al. Casein in the place of beef liver in artificial diets for common carp Cyprinus carpio larvae. Aquat Living Resour v.3, n.3, p.229-234, 1990.

Ciência Rural, v. 31, n. 1, 2001. 